
Revue Revue de l'histoire des religions
del'histoire des religions
$1 \mid 2019$
Corps, ascèse et extinction dans l'histoire du bouddhisme (Inde, Corée, Japon)

\title{
Enrique GARCíA HERnÁN, Ignace de Loyola. Biographie, traduction de Pierre Antoine FABRE
}

Paris, Éditions du Seuil, 2016

Marie Lezowski

\section{(2) OpenEdition}

\section{Journals}

Édition électronique

URL : https://journals.openedition.org/rhr/9667

DOI : $10.4000 /$ rhr.9667

ISSN : 2105-2573

Éditeur

Armand Colin

Édition imprimée

Date de publication : 1 mars 2019

Pagination : 211-213

ISBN : 978-2-200-93230-5

ISSN : 0035-1423

Référence électronique

Marie Lezowski, «Enrique García Hernán, Ignace de Loyola. Biographie, traduction de Pierre Antoine

FABRE », Revue de l'histoire des religions [En ligne], 1 | 2019, mis en ligne le 01 janvier 2021, consulté le

07 janvier 2022. URL : http://journals.openedition.org/rhr/9667 ; DOI : https://doi.org/10.4000/rhr. 9667

Ce document a été généré automatiquement le 7 janvier 2022

Tous droits réservés 


\title{
Enrique GARCÍA HERNÁN, Ignace de Loyola. Biographie, traduction de Pierre Antoine FABRE
}

Paris, Éditions du Seuil, 2016

\author{
Marie Lezowski
}

\section{RÉFÉRENCE}

Enrique GARCíA HERnÁN, Ignace de Loyola. Biographie, traduction de Pierre Antoine FABRE, Paris, Éditions du Seuil, 2016, 566 p., 24 cm, 29 €, ISBN 978-2-02-123165-6.

1 L'histoire d'Ignace de Loyola (v. 1491-1556) a longtemps été l'apanage de la Compagnie de Jésus. Avec cet ouvrage, traduit avec grand soin par Pierre Antoine Fabre, E. García Hernán offre la première biographie de référence écrite par un universitaire non jésuite. Non content de rendre accessible une très riche tradition de travaux, il emprunte une voie inédite pour comprendre le cheminement complexe du fondateur de la Compagnie de Jésus, du charisme à l'institution. De facture classique, le livre suit l'ordre chronologique et n'oublie aucun épisode canonique, tels que la blessure de Pampelune (chap. II, p. 108-124), la vision de Manresa (chap. III, p. 161-163) et le « vœu de Montmartre » (chap. v, p. 268-270). Sous ses atours de vade-mecum, il fait entendre une note très singulière. Il s'émancipe de la légende et met en lumière toutes les incertitudes d'un parcours hésitant. Sans afféteries, l'historien écrit son enquête en miroir des tâtonnements de son personnage, des ambiguïtés des sources ou de leur absence. Le grand sujet du livre est en effet l'énigme d'un homme toujours en mouvement, qui s'épuise en voyages et en recommencements, jusqu'à l'approbation de la Compagnie : soit pendant l'essentiel de sa vie.

2 En avant-propos, l'auteur rappelle les difficultés posées par les récits contemporains, toujours à charge ou à décharge, légende noire ou dorée. Les histoires jésuites, l'Autobiographie (ou Récit du pèlerin) sont des guides trompeurs, expurgés et réécrits 
pour servir les progrès de la Compagnie. Les papiers des nombreux procès intentés à Ignace sont eux aussi sélectifs et censurés. L'historien doit ajuster sa manière d'écrire à une documentation hétérogène, en trois temps principaux : d'abord dense et parcouru d'hypothèses, le récit suit une ligne plus limpide de 1521 à 1540 , quand il peut se fonder sur le Récit, lu avec prudence et confronté à des sources judiciaires et polémiques. Enfin, à partir de 1540, les sources institutionnelles (lettres, textes fondateurs) prennent le relais et permettent à l'historien de donner des mises au point incisives sur les dossiers brûlants à la création de l'Ordre, par exemple ses liens avec les nouveauxchrétiens. Si les objectifs poursuivis gagnent en netteté dans ces années 1540-1550, Ignace lui-même semble s'éloigner, au profit du groupe des compagnons de la première et de la deuxième générations, hommes et femmes, qui font l'objet du passionnant chapitre VIII, et de réalisations collectives qui lui échappent de plus en plus (chap. IX).

Venons au cœur de l'énigme. Qui est Ignace de Loyola? D’abord, un Espagnol. Rome n'intervient qu'au terme d'un long parcours. Íñigo - son nom jusqu'à l'invention romaine d'« Ignace » - est « le Basque de Loyola », natif du Guipúzcoa (chap. I). Inscrit par sa naissance dans des alliances et des luttes de clans, il est le sujet d'une monarchie ibérique en gestation, "entre Moyen Âge et époque moderne » (chap. II). E. García Hernán reconstitue avec minutie ce qu'on peut savoir ou conjoncturer de sa première formation, de ses rencontres et de ses protecteurs. Íñigo n'est pas tant le soldat campé par l'hagiographie qu'un jeune noble formé à la Cour, petit page, puis scribe et comptable au sein de la Trésorerie royale. Apport capital du livre, la compréhension des réseaux espagnols est directement liée à ses voyages incessants, et ce, au-delà de la prime jeunesse. Ainsi, à partir de 1523, quand Ignace, déjà trentenaire, se lance sur les routes d'Europe, entre l'Italie, Jérusalem, la France, les Flandres et l'Angleterre (chap. III-VI), il s'appuie sur des protecteurs, amis et disciples, espagnols surtout. Chaque déplacement est pour lui l'occasion d'élargir le cercle de ses soutiens, par exemple quand il se rend dans les Flandres (1529) et en Angleterre (1531), pour collecter les fonds nécessaires à ses études (chap.v). Retraçant le fameux séjour d'Ignace à Paris, de 1528 à 1535, E. García Hernán évoque autour de lui, en des pages mémorables, toute la communauté espagnole du Quartier latin, divisée dans ses rapports avec les réformés et les spirituels. Cette relecture espagnole, très originale, vaut pour les dernières années romaines; Ignace, gagné par un « retour aux sources familiales» (p. 474), encourage l'alliance entre les Borja et les Loyola. Grand connaisseur de la noblesse hispanique, E. García Hernán nuance donc la rupture d'Ignace avec son lignage, mise en avant par l'hagiographie (p. 279 sqq.). C'est à la parenté élargie, définie par l'alliance, qu'il se réfère pour comprendre la famille ignatienne. Il éclaire ainsi la capacité de la Compagnie à se développer en ralliant oncles et neveux, ou toute une fratrie. Ignace cultive ces adhésions familiales en rendant visite aux parents de ses premiers compagnons (p. 458-459).

La deuxième clé de lecture offerte par le livre - la plus importante - est une «manière de procéder", un " "tempérament" fait de souplesse et de patience» (p. 489). Spécialiste d'histoire diplomatique et militaire, E.García Hernán entend écrire la "biographie d'un homme concret», d'un "médiateur [...] réconciliateur des adversaires, des mariés malheureux, des ennemis mortels, des idées opposées, des doctrines douteuses et des croyances impossibles » (p. 33). Comprendre le don d'Ignace pour la conciliation suppose de donner une vue d'ensemble de ses fréquentations: l'auteur traque sans relâche le plus grand nombre possible de ses contemporains, 
insignes ou obscurs, qui, entre $\mathrm{xV}^{\mathrm{e}}$ et $\mathrm{xVI}{ }^{\mathrm{e}}$ siècle, vivent le renforcement des administrations monarchiques, le basculement du foisonnement spirituel à la ContreRéforme. L'historien voit cette "manière de procéder » dans la formation continue de groupes de compagnons, dans les liens maintenus à distance, par lettres, mais encore dans la forme des Exercices spirituels, analysés comme une négociation ouverte par l'exercitant entre lui-même et sa propre vie (p.490). Ce talent à fédérer est constamment à l'œuvre, y compris quand Ignace ne sait pas encore comment il souhaite suivre le Christ. À Barcelone et Alcalà, il gagne ses premiers "partisans » en dispensant des conseils et en commençant à donner les Exercices (chap. IV). Des années plus tard, à Rome, Ignace emploie ce don, aiguisé par des années de pratique, pour s'employer à sauver le couple malheureux formé par Marguerite de Parme et Ottavio Farnèse, sans s'aliéner l'Espagne ni le pape (p. 356-368). La négociation à haut risque est narrée à renfort de métaphores militaires, chères à l'auteur (Ignace tâchant de " passer entre les balles»!), mais c'est bien un conseil spirituel que propose Ignace, un temps confesseur de Marguerite.

5 Pour E. García Hernán, Ignace peut être compris par ses actes. Ses préoccupations spirituelles lui échappent souvent. Cependant, loin de se résumer à une collection de faits positifs, le récit progresse souvent par conjonctures. Il retrace de nombreuses rencontres possibles, singulièrement quand il décrit le milieu mystique espagnol dans lequel Ignace accomplit sa conversion, à partir de 1521 (chap. II et III). Immergé dans un monde de béates et de mystiques, et peu après sa rencontre probable avec la visionnaire María de Santo Domingo, Ignace adopte un mode de vie alumbrado, des façons de prier, de se vêtir, de prêcher, de guider sans formation préalable.

D'où le soupçon d'alumbradismo, qui poursuit Ignace sa vie durant. Cette accusation rejoint une interrogation cardinale de l'historien: quelle est la source d'inspiration d'Ignace quand il prêche, qu'il donne les Exercices? Une lecture serrée des procès qui lui sont intentés entre 1526 et 1538 permet à l'auteur de démêler les arguments de l'accusation et les moyens mis en œuvre par Ignace pour sa défense. En Espagne, à Paris, comme en Italie, Ignace est suspect de mysticisme parce qu'il prêche et qu'il conseille sans avoir reçu de formation universitaire. Il est sommé de s'expliquer et interdit de prêcher jusqu'à l'obtention de grades. Face à ces accusations répétées, il n'intègre pas un ordre existant - ce qui serait le choix le plus simple - mais négocie avec les autorités ecclésiastiques, en finissant par suivre des études régulières à Paris et en œuvrant à la naissance d'une nouvelle religion de prêtres réguliers. Comme le montrent les chapitres IV à VII, cette mise en conformité est lente et tortueuse, en particulier parce qu'Ignace tient à préserver la liberté de mouvement de ses compagnons. Il obtient peu à peu la reconnaissance d'une nouvelle catégorie de clerc, qui étudie et prêche sans être fixé à un lieu. L'invention de ce statut singulier est explicitée avec acuité par la propre expérience d'Ignace, longtemps déchiré entre le désir (ou l'obligation) de se former et celui de se gagner des compagnons (p. 237), entre la sédentarité de l'étude et les voyages guidés par le secours spirituel.

7 Espagnol itinérant, négociateur sur la corde raide, alumbrado présumé : tel est en peu de mots le portrait d'Ignace de Loyola brossé par E. García Hernán, un portrait neuf et frémissant, qui vient bousculer l'histoire routinière et ressusciter le charisme d'un homme. Un nouveau classique. 


\section{AUTEURS}

MARIE LEZOWSKI

Université d'Angers. 